\title{
Comparison of the results and complications of palmar and dorsal miniinvasive approaches in the surgery of scaphoid fractures. A prospective randomized study
}

\author{
Pavel Drac ${ }^{\mathrm{a}}$, Igor Cizmara , Pavel Manaka, Jan Hrbek ${ }^{\mathrm{b}}$, Michal Reskac, Pavel Filkuka ${ }^{\mathrm{d}}$, Jana Zapletalova ${ }^{\mathrm{e}}$
}

\begin{abstract}
Aims. To compare the functional results and complications associated with palmar percutaneous and dorsal limited approaches in the surgical treatment of nondisplaced or minimally displaced scaphoid fractures type B2.

Methods. A total of 76 patients with acute nondisplaced or minimally displaced type B2 scaphoid fractures were included in a prospective randomised study. The assignment patients to groups according to type of operative approach was based on systematic sampling. Clinical follow-up, X-ray or CT imaging were performed at four, eight and twelve weeks and one year after the surgery. Patient satisfaction and the results of DASH form were evaluated as well. The differences were statistically tested.

Results. We found significantly better flexion and grip strength in the group of palmar percutaneous aprroach during the follow-up examination at eight weeks after the surgery. Significantly better flexion of the same group persisted at twelve-week follow-up examination. No differences in results or complications were statistically significant up to one year follow up.

Conclusions. We found no advantage to the palmar percutaneous approach in the treatment of nondisplaced and minimally displaced scaphoid fractures type B2 compared to dorsal limited approach.
\end{abstract}

Key words: scaphoid fractures, palmar percutaneous approach, dorsal limited approach, functional results, complications

Received: January 29, 2012; Accepted: June 6, 2012; Available online: November 12, 2012

http://dx.doi.org/10.5507/bp.2012.060

a Department of Traumatology, University Hospital Olomouc, Czech Republic
${ }^{b}$ Department of Radiology, University Hospital Olomouc
cIst Clinic of Surgery, St. Anne's University Hospital Brno
${ }^{d}$ Department of Radiology, St. Anne's University Hospital Brno
eDepartment of Medical Biophysics, Faculty of Medicine and Dentistry, Palacky University Olomouc
Correspondig author: Pavel Drac, e-mail: dracpa@seznam.cz

\section{INTRODUCTION}

Scaphoid fractures affect mainly young men. Missed or late diagnosis can prolong the healing period or lead to nonunion and subsequent degenerative changes (SNAC wrist), which requires additional surgery. The treatment results do not very often fulfill the patient's and surgeon's expectations. Recently, there has been an increased trend toward surgical fixation using conventional or miniinvasive approaches based on the type of scaphoid fractures ${ }^{1,2}$. Surgery is fully indicated for scaphoid fractures type B1-B5 according to the Herbert classification ${ }^{3}$ (unstable fractures). Surgical treatment provides for early wrist physiotherapy, better functional results and shorter sick leave ${ }^{4-8}$. The total treatment costs are similar to those of conservative treatment - as shown in the economic analys ${ }^{9,10}$. Complete nondisplaced and minimally displaced fractures of the scaphoid waist (middle third of scaphoid) can be treated using dorsal or palmar miniinvasive approaches. Both methods have pros and cons. Recent studies on results and complications of both surgical methods are available ${ }^{11}$. The main limitations of studies which compare palmar and dorsal approaches include their retrospective method and relatively small samples ${ }^{12-14}$.

\section{MATHERIALS AND METHODS}

Only patients with acute nondisplaced or minimally displaced type B2 scaphoid waist fractures (according to Herbert's classification ${ }^{3}$ ) were included in this prospective randomised study. Minimal displacement refers to a longitudinal distraction of fragments up to $2 \mathrm{~mm}$ with no lateral or rotational distraction on CT scans. There were 76 patients who met these criteria, agreed to and signed the informed content form from July 2008 to December 2010. Patients with complicated fractures such as transscaphoid perilunate dislocations and patients with associated fractures of other carpal bones or distal radius were excluded. Inclusion of patients into groups (group A - palmar percutaneous approach, group B - dorsal limited approach) was based on systematic sampling. This provided system homogeneity in age, sex and dominance of the injured extremity.

Both techniques - the palmar percutaneous and dorsal limited approaches - have been repeatedly and closely described in the literature ${ }^{1,6,15,16}$. All surgeries were performed under general anaesthesia with a pneumatic tourniquet applied to the upper arm. Osteosynthesis was performed by the trauma surgeons involved in this proj- 
ect (P.D., I.Č., M.R.) using cannulated headless screws (Herbert screws) up to 14 days after the trauma.

Using palmar percutaneous approach, an 8-10 $\mathrm{mm}$ transverse skin incision was made $10 \mathrm{~mm}$ distal to the scaphotrapezial joint and blunt dissection down to the bone without opening the capsule of the scaphotrapezial joint was performed. A towel roll was used under the supinated wrist to allow for adequate wrist extension. With the aid of image intensifier a guide wire was inserted into the bone through the tubercle, and passed centrally down the scaphoid. A cannulated screw was then inserted over the guide wire to be fully buried within the bone. After removal of the guide wire the wound was closed with one or two skin sutures.

In cases involving the dorsal approach, a $15-20 \mathrm{~mm}$ longitudinal skin incision was made starting over the Lister tubercle and heading toward the base of the $3^{\text {rd }}$ metacarpal bone. The extensor pollicis longus tendon was displaced radially. The longitudinal capsulotomy was performed and the wrist was fully flexed. The guide wire was inserted to the scaphoid through the proximal pole closely to the origin of scapho-lunate ligament. The position of the guide wire was checked by image intensifier and the screw was inserted over the wire to be fully sunk beneath the proximal surface of the scaphoid. Removing guide wire, the capsule and skin were closed with sutures.

All the operated wrists were then postoperatively fixed in a removable splint for a period of two weeks and, after stitches removal and physiotherapy was started.

Two patients (one patient from each group) were lost to follow-up. The remaining 74 patiens were evaluated as there were data from all scheduled follow-up examinations. Clinical follow-up was performed four, eight and twelve weeks after the surgery, X-ray examination after four weeks and CT imaging twelve weeks after the surgery according to a standardized protocol. In case of the clinical or radiologic sign of delayed union, the follow-up continued with the clinical and radiological examinations every six weeks. The final clinical, X-ray and CT examinations were performed twelve months postoperatively. We evaluated the range of motion (flexion, extension, radial and ulnar deviation), grip strength using the Jamar dynamometer (as a percentage of the opposite, unaffected wrist), the presence of persisting complaints, pain during sports and physical activities. During X-ray and CT imaging we evaluated the presence of screw protrusion over subchondral bone and presence of degenerative changes of the wrist. Software Web 1000 4.1. (AGFA) was used for this measurement. We evaluated the incidence of complications in each group. The complications which needed surgery included: nonunion, malunion, fracture, compartment syndrome, septic arthritis, nonreversible trauma to the sensory nerve branches, vessel injury, complex regional pain syndrome, tendon rupture and complications which needed nonsurgical treatment: superficial infection, secondary wound healing, transitory nerve injury, tendon irritation. All the patients were asked about their satisfaction with the outcome (satisfied/dissatisfied) and all filled in the DASH form ${ }^{17}$. The differences between the group outcomes were statistically tested by the Student's t-test, the Sign test and the Mann-Whitney U-test. Any differences were considered statistically significant when $P<0.05$. The SPSS 15 (SPSS Inc., Washington, USA) professional software was used for the statistical analysis.

\section{RESULTS}

The compared groups were homogenous according to age, gender and hand dominance (Table 1). We found one symptomatic scaphoid nonunion in the group A (palmar percutaneous approach). There were no statistically significant differences between the two groups with respect to flexion, extension, radial and ulnar deviation, grip strength, presence of persisiting complaints, patient satisfaction or DASH score during the follow-up examination twelve months after the surgery as shown in Table 2. The differences between groups for complications were also not significant as shown in Table 3. Comparing the

Table 1. Assessment of the homogeneity of group A (palmar percutaneous approach) and group B (dorsal limited approach).

\begin{tabular}{lccc}
\hline & Group A & Group B & $P$ \\
\hline Age (years) & 31.6 & 29.2 & 0.141 \\
$\begin{array}{l}\text { Gender (percent- } \\
\text { age of women) }\end{array}$ & 8.1 & 13.5 & 0.711 \\
$\begin{array}{l}\text { Hand dominance } \\
\text { (percentage of } \\
\text { dominant hand }\end{array}$ & 56.8 & 51.3 & 0.816 \\
injury)
\end{tabular}

Table 2. Functional outcome during the follow-up examination at one year after surgery. Flexion, extension, radial and ulnar deviation and grip strength compared with the opposite, unaffected wrist.

Complaints, pain and satisfaction (yes/no) as a percentage of each group. Group A (palmar percutaneous approach), group B (dorsal limited approach).

\begin{tabular}{lccc}
\hline & Group A & Group B & $P$ \\
\hline Flexion* & 93.7 & 89.4 & 0.108 \\
Extension* & 92.5 & 95.0 & 0.301 \\
Radial deviation* & 92.5 & 94.7 & 0.626 \\
Ulnar deviation* & 100.0 & 94.1 & 0.496 \\
Grip strength* & 93.8 & 94.1 & 0.626 \\
Free of persistent & 70.3 & 64.9 & 0.804 \\
complaints* & & & \\
Resting pain* & 0 & 2.7 & 1.000 \\
Pain during activities* & 29.7 & 32.4 & 1.000 \\
DASH score & 3.89 & 3.70 & 0.740 \\
Patient satisfaction* & 97.3 & 97.3 & 1.000 \\
\hline
\end{tabular}

* The values are given in per cent. 
Table 3. Comparison of the complications in groupA (palmar percutaneous approach) and group B (dorsal limited approach).

\begin{tabular}{lccc}
\hline & Group A & Group B & $P$ \\
\hline $\begin{array}{l}\text { Wound infection } \\
\begin{array}{l}\text { Guidewire bending } \\
\text { / breakage }\end{array}\end{array}$ & 1 & 1 & 1.000 \\
$\begin{array}{l}\text { Transient hypo- } \\
\text { paresthesia }\end{array}$ & 0 & 2 & 0.615 \\
$\begin{array}{l}\text { Screw protrusion } \\
\text { or malposition }\end{array}$ & 2 & 0 & 0.493 \\
$\begin{array}{l}\text { Non-union } \\
\text { Total complications }\end{array}$ & 1 & 0 & 1.000 \\
\hline
\end{tabular}

Table 4. Functional outcome during the follow-up examination at four, eight and twelve weeks after surgery.

Flexion, extension, radial and ulnar deviation and grip strength compared with the opposite, unafected wrist. Complaints, pain and satisfaction (yes/no) as a percentage of each group. Group A (palmar percutaneous approach), group B (dorsal limited approach).

\begin{tabular}{lrrr}
\hline & Group A & Group B & \multicolumn{1}{c}{$P$} \\
\hline & & & \\
\hline 4-weeks follow-up examination & & 32.4 & 0.100 \\
\hline $\begin{array}{l}\text { Free of persistent com- } \\
\text { plaints* }\end{array}$ & 54.1 & & \\
Resting pain* & 5.4 & 10.8 & 0.674 \\
Pain during & 40.5 & 56.8 & 0.245 \\
activities* & & & \\
\hline 8-weeks follow-up examination & & & \\
\hline Flexion* & 89.7 & 72.3 & $<0.0001$ \\
Extension* & 79.7 & 83.0 & 0.284 \\
Radial deviation* & 83.1 & 80.0 & 0.714 \\
Ulnar deviation* & 87.7 & 84.2 & 0.237 \\
Grip strength* & 82.8 & 75.2 & 0.031 \\
\hline 12-weeks follow-up examination & & & \\
\hline Flexion* & 91.5 & 81.6 & 0.0003 \\
Extension* & 86.2 & 90.1 & 0.97 \\
Radial deviation* & 89.8 & 87.4 & 0.857 \\
Ulnar deviation* & 90.0 & 88.0 & 0.373 \\
Grip strength* & 91.0 & 87.7 & 0.434 \\
Free of persistent com- & 64.9 & 51.4 & 0.346 \\
plaints* & & & \\
Resting pain* & 2.7 & 8.1 & 0.615 \\
Pain during & 32.4 & 40.5 & 0.630 \\
activities* & & & \\
DASH score & 5.75 & 5.29 & 0.495 \\
\hline
\end{tabular}

*The values are given in per cent. data obtained during the follow-up examination four, eight and twelve weeks following surgery we found significant differences only in flexion at $8^{\text {th }}$ - and $12^{\text {th }}$-week follow-up examination and grip strength during $8^{\text {th }}$-week follow-up examination in favour of group A (palmar percutaneous approach). See Table 4.

\section{DISCUSSION}

Nondisplaced and minimally displaced fractures of the scaphoid waist (the middle third of the scaphoid) can be treated using the dorsal or palmar miniinvasive approaches. Both methods have their pros and cons.

The dorsal approach has been reported by deMaaggd in 1989 for the treatment of nonunions of the proximal pole of the scaphoid ${ }^{15,16}$. Anatomical and clinical studies $^{14,18}$ showed that the dorsal techniques resulted in a centered screw throughout the scaphoid, whereas the screws inserted palmarly were centered in the waist and proximal pole but not in the distal pole. The centered screw leads to better rigidity and stability of the osteosynthesis ${ }^{19}$. The disadvantage of the dorsal approach is the necessity for extensor retinaculum partial release and the violation of radioscaphoid joint by the drill. In contrast, this approach enables the screw to be sunk underneath the bone cartilage to prevent distal radius cartilage irritation in case of screw protrusion ${ }^{20}$.

On the other hand, using the dorsal percutaneous approach, the surgeon has to fully rely on fluoroscopic help without seeing the screw sink and fracture reduction. ${ }^{11,14}$. Arthroscopic assistance can solve this problem ${ }^{13}$ but the procedure is demanding in terms of equipment and experience. Additionally, an anatomic study by Adamani et al. ${ }^{21}$ showed a high risk of tendon and nerve damage in the percutaneous fixation of the scaphoid through the dorsal approach. In $58 \%$ of wrist cadavers they found the guidewire going through the extensor pollicis longus tendon or extensor indicis proprius tendon or extensor digitorum communis to the index, and in $42 \%$ the guide wire passed through the terminal portion of the posterior interosseous nerve.

Palmar percutaneous screw fixation of scaphoid fractures was first described by Streli ${ }^{22}$. In contrast to dorsal approaches, the palmar percutaneous approach has no risk of tendon or nerve structure damage. In an effort to provide perpendicular direction of the guide wire to the fracture line, some surgeons insert the wire through trapezium or perform a partial resection of the trapezium ${ }^{23}$. This method involves risk of later carpometacarpal or scaphotrapezial arthrosis development ${ }^{24,25}$.

The above facts justify our choice of the palmar percutaneous and dorsal limited approaches for the surgical treatment of nondislocated or minimally dislocated scaphoid waist fractures. Soubeyrand et al. ${ }^{26}$ demonstrated the suitability of both approaches for the treatment of B2 type fractures of the scaphoid using a computer model in cadavers.

We found one symptomatic scaphoid nonunion in group A (palmar percutaneous approach) but it was not 
statistically significant $(P=1.000)$. The patient refused a second surgery because of his moderate symptoms. Similarly, in the three published studies comparing the dorsal and palmar approaches for the scaphoid fracture treatment ${ }^{12-14}$, the union rate for the palmar approach group was $80-100 \%$ and for the dorsal approach group 81-100\% and no statistical differences were found between groups.

In our study there were no statistically significant differences between the two groups with respect to flexion, extension, radial and ulnar deviation, grip strength, presence of persisiting complaints and patient satisfaction during the follow-up examination at twelve months after the surgery. Jeon et al. ${ }^{14}$ and Slade et al. ${ }^{13}$ reported no statistical differences in flexion, extension, radial and ulnar deviation with the average follow-up of 30 and 18 months, respectively. Only Polsky et al. ${ }^{12}$ found significantly less ulnar deviation $(P<0.01)$ in the dorsal approach patients $\left(25.9^{\circ}\right)$ compared with palmar approach patients $\left(40.4^{\circ}\right)$.

Jeon et al. ${ }^{14}$ used the Mayo wrist score for the functional assessment at an average 30 months follow-up and found no significant differences ( 96 points for the dorsal approach group and 95 points for the palmar approach group). We used the DASH score and found 3.70 (out of 100) for the palmar approach group and 3.89 for the dorsal approach group $(P=0.740)$. Bedi et al. ${ }^{20}$ reffered the final DASH score of 6.12 after the average of 98 weeks of follow-up for the analogous patient group using the limited dorsal approach.

Comparing the presence of persisting complaints, resting pain and pain during activities, we found slightly more patients free of persistent complaints in group A (palmar percutaneous approach). The outcomes of these studies using unspecified pain scale ${ }^{14}$ and visual analog scale ${ }^{12}$ were analogous $(P=0.910, P=0.759)$.

The overall rates of complications were $13.5 \%$ for group A and $16.2 \%$ for group B $(P=1.000)$. Analyzing our complications we realized the majority of them were caused by technical error during surgery (guidewire bending or breakage, screw protrusion or malposition). From the recent literature the reported total complication rates of between $0 \%$ and $29 \%$ for the palmar and dorsal miniinvasive approaches ${ }^{11-14,20}$. However, it is necessary to realize that the definition of complication varies.

We found no studies comparing the functional results of the surgically treated scaphoid fractue using the palmar or dorsal miniinvasive approaches during the first three months postoperatively. We understand our finding of the significantly better flexion in the palmar approach group (89.7\% of the opposite unaffected wrist flexion compared to $72.3 \%$ in the dorsal approach group in the eight-week follow-up $(P<0.0001)$, lasting to the twelve-week followup $(91.5 \%$ against $81.6 \%, P=0.0003)$ to be probably due to the immature scar at the site of the dorsal approach. It can also explain the better grip strength in the dorsal approach group (82.8\% compared to $75.2 \%$ ).

\section{CONCLUSIONS}

Based on a twelve-month follow-up in this prospective randomised study, we did not find the palmar percutaneous approach for the treatment of acute nondisplaced or minimally displaced type B2 scaphoid fractures to be superior to the dorsal limited approach. The functional results and complication rate were comparable. We found the majority of the encountered complications to have been caused by technical error during the surgery.

\section{ACKNOWLEDGEMENTS}

This article has been supported by grant project IGA MZCR NS 9623-4/2008.

\section{CONFLICT OF INTEREST STATEMENT}

The authors state that there are no conflicts of interest regarding the publication of this article.

\section{REFERENCES}

1. Bond CD, Shin AY. Percutaneous cannulated screw fixation of acute scaphoid fractures. Tech Hand Up Extr Surg 2000;4:81-7.

2. Moser VL, Krimmer H, Herbert TJ. Minimal invasive traeatment for scaphoid fractures using the canulated Herbert screw system. Tech Hand Up Extr Surg 2003;7:141-6.

3. Herbert TJ.The fractured scaphoid. St Louis: Quality Medical Publishing, Inc; 1990.

4. Arora R, Gschwentner M, Krappinger D, Lutz M, Blauth M, Gabl M. Fixation of nondisplaced scaphoid fractures: making treatment cost effective. Prospective controlled trial. Arch orthop Trauma Surg 2007; 127:39-46.

5. Dias JJ, Dhukaram V, Abhinav A, Bhowal B, Wildin CJ. Clinical and radiological outcome of cast immobilisation versus surgical treatment of acute scaphoid fractures at a mean follow-up of 93 months. J Bone Joint Surg 2008;90B:899-905.

6. Dráč $P$, Maňák $P$, Labónek I. Percutaneous osteosynthesis versus cast immobilisation for the treatment of minimally and non-displaced scaphoid fractures. Functional outcomes after a follow-up of at least 12 month. Biomed Papers 2005;149:149-51.

7. McQueen MM, Gelbke MK, Wakefield A, Will EM, Gaebler C. Percutaneous screw fixation versus conservative treatment for fractures of the waist of the scaphoid: a prospective randomised study. J Bone Joint Surg 2008;90B:66-71.

8. Vinnars B, Pietreanu M, Bodestedt A, Ekenstam F, Gerdin B. Nonoperative treatment of acute scaphoid fractures. A randomized clinical trial. J Bone Joint Surg 2008;90A:1176-85.

9. Dráč $P$, Maňák P. An economic analysis of scaphoid fracture treatment. Eur J Trauma 2006; 32, Suppl 1:188.

10. Vinnars B, Ekenstam FA, Gerdin B. Comparison of direct and indirect costs of internal fixation and cast treatment in acute scaphoid fractures: a randomized trial involving 52 patients. Acta Orthop 2007;78:672-9.

11. Bushnell BD, McWilliams AD, Messer TM.Complications in dorsal percutaneous cannulated screw fixation of nondisplaced scaphoid waist fracture. J Hand Surg 2007; 32A:827-33.

12. Polsky MB, Kozin SH, Porter ST, Thoder JJ. Scaphoid fractures: dorsal versus volar approach. Orthopedics, 2002;25:817-9.

13. Slade J, Lozano-Calderon S, Merrell G, Ring D. Arthroscopic-assisted percutaneous reduction and screw fixation of displaced scaphoid fracture. J Hand Surg 2008;33E:350-4. 
14. Jeon IH, Micic ID, Oh CW, Park BC, Kim PT. Percutaneous screw fixation for scaphoid fracture: A comparison between the dorsal and the volar approaches. J Hand Surg 2009;34A:228-36.

15. DeMaadg RL, Engber WD. Retrograde Herbert screw fixation for treatment of proximal pole scaphoid nonunions. J Hand Surg 1989; 14A:996-1003.

16. Martus JE, Bedi A, Jebson PJL. Cannulated variable pitch compression screw fixation of scaphoid fractures using a limited dorsal approach. Tech Hand Up Extr Surg 2005;9:202-6.

17. Hudak PL, Amadio PC, Bombardier C. Development of an upper extremity outcome measure: the DASH (disabilities of the arm, shoulder and hand) (corrected). The Upper Extremity Collaborative Group (UECG). Am J Ind Med 1996;29:602-8.

18. Chan KW, McAdams TR. Central screw placement in percutaneous screw scaphoid fixation: a cadaveric comparision of proximal and distal techniques. J Hand Surg 2004; 29A:74-9.

19. McCallister WV, Knight J, Kaliappan R, Trumble TE. Central placement of the screw in simulated fractures of the scaphoid waist: a biomechanical study. J Bone Joint Surg 2003; 85A:72-7.

20. Bedi A, Jebson PJL, Hayden RJ, Jacobson JA, Martus JE.Internal fixation of acute, nondisplaced scaphoid waist fracture via a limited dorsal approach: an assessment of radiographic and functional outcomes. J Hand Surg 2007; 32A:326-33.
21. Adamani DC, Mikola EA, Fraser BJ. Percutaneous fixation of the scaphoid through a dorsal approach: an anatomic study. J Hand Surg 2008; 33A:327-31.

22. Streli R. Perkutane Verschraubung des Handkahnbeines mit Bohrdraht-Kompressionsschraube. Zentralbl Chir 1970;95:1060-78.

23. Tu YK, Chen AC, Chou YC, Ueng SWN, Ma CH, Yen CY. Treatment for scaphoid fracture and nonunion - the aplication of $3.0 \mathrm{~mm}$ cannulated screws and pedicle vascularized bone grafts. Injury 2008;39:S96106.

24. Saedén B, Törnkvist $H$, Ponzer $S$, Hoglund M. Fracture of the carpal scaphoid. A prospective, randomised 12-year follow-up comparing operative and conservative treatment. J Bone Joint Surg 2002;83B:230-4.

25. Nicholl JE, Buckland-Wright JC Degenerative changes at the scaphotrapezial joint following Herbert screw insertion: A radiographic study comparing patients with scaphoid fracture and primary hand arthritis. J Hand Surg 2000;25B:422-6.

26. Soubeyrand M, Biau D, Mansour C, Mahjoub S, Molina V, Gagey O. Comparison of percutaneous dorsal versus volar fixation of scaphoid waist fractures using a computer model in cadavers. J Hand Surg 2009;34A:1838-44. 